\title{
Mixed matrix formulations with MOF molecular sieving for key energy-intensive separations
}

Gongping Liu, 1,2

Valeriya Chernikova, 3

Yang Liu, 1

Kuang Zhang, 1

Youssef Belmabkhout, 3

Osama Shekhah, 3

Chen Zhang, 1

Shouliang Yi, 1

Mohamed Eddaoudi, 3

Emailmohamed.eddaoudi@kaust.edu.sa

William J. Koros, $1 \square$

Emailwjk@chbe.gatech.edu

1 School of Chemical \& Biomolecular Engineering, Georgia Institute of Technology, Atlanta, GA, USA

2 State Key Laboratory of Materials-Oriented Chemical Engineering, Nanjing Tech University, Nanjing, China 
3 Advanced Membranes \& Porous Materials Center, Division of Physical Sciences and Engineering, Functional Materials Design, King Abdullah University of Science and Technology, Discovery and Development research group (FMD3), Thuwal, Saudi Arabia

Received: 18 September 2017 / Accepted: 14 December 2017

\section{Abstract}

Membrane-based separations can improve energy efficiency and reduce the environmental impacts associated with traditional approaches. Nevertheless, many challenges must be overcome to design membranes that can replace conventional gas separation processes. Here, we report on the incorporation of engineered submicrometre-sized metalorganic framework (MOF) crystals into polymers to form hybrid materials that successfully translate the excellent molecular sieving properties of face-centred cubic (fcu)-MOFs into the resultant membranes. We demonstrate, simultaneously, exceptionally enhanced separation performance in hybrid membranes for two challenging and economically important applications: the removal of $\mathrm{CO}_{2}$ and $\mathrm{H}_{2} \mathrm{~S}$ from natural gas and the separation of butane isomers. Notably, the membrane molecular sieving properties demonstrate that the deliberately regulated and contracted MOF pore-aperture size can discriminate between molecular pairs. The improved performance results from precise control of the linkers delimiting the triangular window, which is the sole entrance to the fcu-MOF pore. This rational-design hybrid approach provides a general toolbox for enhancing the transport properties of advanced membranes bearing molecular sieve fillers with sub-nanometre-sized pore-apertures.

$\mathrm{AQ1}$

$\mathrm{AQ2}$

AQ3

Submicrometre MOF particles are incorporated into polymers to form mixed matrix membranes. Molecular sieving enables performance far beyond current limits for two applications, butane isomer separation and combined $\mathrm{CO}_{2} / \mathrm{H}_{2} \mathrm{~S}$ 
removal from natural gas.

\section{Main}

Gas separations are essential processes in the energy and chemical industries[1, 2], typified by natural gas purification and butane isomer separation. Raw natural gas typically contains acid gas components, and must be purified by amine scrubbing to meet pipeline specifications $\left(<2 \% \mathrm{CO}_{2}\right.$ and $\left.<4 \mathrm{ppm}_{2} \mathrm{~S}\right)[3]$. Moreover, $n$-butane must be separated from isobutane by distillation prior to the production of high-octane gasoline blending stock[4]. Gas separation membranes, although widely used for hydrogen recovery and air separation, face challenges for the above-mentioned energy-intensive processes[5]. Existing high-performance membrane materials for $\mathrm{CO}_{2} / \mathrm{CH}_{4}$ separation show much lower efficiency in $\mathrm{H}_{2} \mathrm{~S}$ removal due to the closer molecular kinetic diameter $\left(d_{k}\right)$ of $\mathrm{H}_{2} \mathrm{~S}(3.6 \AA)$ than $\mathrm{CO}_{2}(3.3 \AA)$ to $\mathrm{CH}_{4}(3.8 \AA)$, which hinders the attainment of an ultralow $\mathrm{H}_{2} \mathrm{~S}$ concentration for pipeline transportation. For separating butane isomers with similar physico-chemical properties, existing pure polymer membranes are economically unattractive due to their low permeability or selectivity[6], whereas zeolite (for example, MFI, a type of aluminosilicate zeolite) membranes show high permeability and selectivity[7] but face difficulties in economical and scalable fabrication.

AQ4

AQ5

A shift to a hybrid mixed-matrix approach based on dispersing highly selective and permeable molecular sieve particles in a processable polymer matrix is an exciting platform for advanced membrane materials[8]. The approach can provide a highly desirable membrane with both high separation performance assisted by high-performing molecular sieves, and economical fabrication based on a processing approach similar to that for simple, pure polymeric membranes[2]. Matching the transport properties and interphase compatibility between a given molecular sieve and polymer is fundamental to create hybrid mixed-matrix membranes with attractive performance. It is to be noted that inorganic zeolites generally show poor compatibility with organic polymers, and often require additional surface modification to avoid non-selective interfacial voids[9, 10, 11]. Metal-organic frameworks (MOFs) consisting of metal ions/clusters connected by organic linkers represent another important class of crystalline porous materials with potential molecular sieving properties for gas 
separations[12, 13, 14, 15], owing to the relatively facile tunability of their pore-aperture size and pore system[16, 17]. Appropriately, numerous high-performance pure MOF membranes and MOF hybrid membranes have been developed for hydrogen[18], $\mathrm{CO}_{2}[19,20,21,22]$ and olefin/paraffin separations[23, 24, 25, 26]. Recently, MOF adsorbents have shown unprecedented $\mathrm{H}_{2} \mathrm{~S} / \mathrm{CO}_{2} / \mathrm{CH}_{4}$ separation properties via an equilibrium-based adsorption mechanism[27, 28] and $n \mathrm{C}_{4} / i \mathrm{C}_{4}$ separation via a molecular sieving adsorption mechanism[27, 29].

Despite these successes, MOF-based membranes have rarely been reported for $\mathrm{H}_{2} \mathrm{~S}$ removal from natural gas or for butane isomer separation, partially due to the difficulty in handling, testing and scale-up of pure MOF membranes, the stability of MOFs to $\mathrm{H}_{2} \mathrm{~S}$, the limited number of MOFs with reported molecular sieving behaviour, and the excessively large and/or flexible apertures in most MOFs[30]. Although some zeolitic imidazolate framework (ZIF) materials having small poreaperture size $(<3.8 \AA)$ might be suitable for discriminating small gases from $\mathrm{CH}_{4}$, the structural stability in highly corrosive $\mathrm{H}_{2} \mathrm{~S}$ remains problematic for such standard ZIFs[31]. Other well-studied MOFs with apertures $>7 \AA$ (for example, HKUST1[32], MIL-53[33], MOF-5[34] and MOF-74[35]) are unable to show substantial molecular sieving properties for $n$-butane $\left(n \mathrm{C}_{4}, d_{k}: 4.5 \AA\right)$ and iso-butane $\left(i \mathrm{C}_{4}, d_{k}: 5.0 \AA\right)$ separation.

Here we report a new type of hybrid membrane derived from a rare-earth (RE) MOF with a face-centered cubic (fcu) topology (RE-fcu-MOF), with interconnected tetrahedral and octahedral cages whose faces comprise a triangular window - the sole entrance to the MOF pore system for guest molecules[36] (Fig. 1a). The size and circumference of the triangular pore-aperture are precisely constrained by the length, bulkiness and local movement of the encompassed di-topic linker (1,4-naphthalenedicarboxylate (naph)[37] or fumarate (fum)[29] studied here), conferring the pore system entrance with the desired molecular sieving capabilities appropriate for natural gas purification and butane isomer separation. The fine-tuned RE-fcu-MOF (where RE is Y for the fum linker or Eu for the naph linker) adsorbents offer molecular-sieving-based separations, for example, $n \mathrm{C}_{4} / i \mathrm{C}_{4}[29]$, and their subsequent introduction as engineered submicrometre-sized crystals into rational-selected polymers yields highly enhanced membrane molecular sieving properties for the challenging $\mathrm{H}_{2} \mathrm{~S} / \mathrm{CO}_{2} / \mathrm{CH}_{4}$ and $n \mathrm{C}_{4} / i \mathrm{C}_{4}$ separations.

\section{Fig. 1}

Design and fabrication of RE-feu-MOF/polymer hybrid membranes. 
a, Schematic of designing the hybrid membrane based on incorporating RE-fcu-MOF molecular sieve crystals into 6FDADAM polymer matrix. By varying the length and bulkiness of the linker, the triangular pore-aperture of RE-fcu-MOFs can be finely tuned for selective diffusion of $\mathrm{H}_{2} \mathrm{~S} / \mathrm{CO}_{2} / \mathrm{CH}_{4}$ or $n \mathrm{C}_{4} / i \mathrm{C}_{4}$ gas pairs. $\mathbf{b}-\mathbf{f}$, Scanning electron microscope images of assynthesized (b) and cryogenic-grinding/sedimentation treated (c) Y-fum-fcu-MOF crystals, cross-sectional Y-fum-fcuMOF/6FDA-DAM hybrid membranes dense film with $17.8 \mathrm{wt} \%$ MOF loading (d,e) and asymmetric hollow fibre with 13.0 wt\% Y-fum-fcu-MOF loading (f,g).
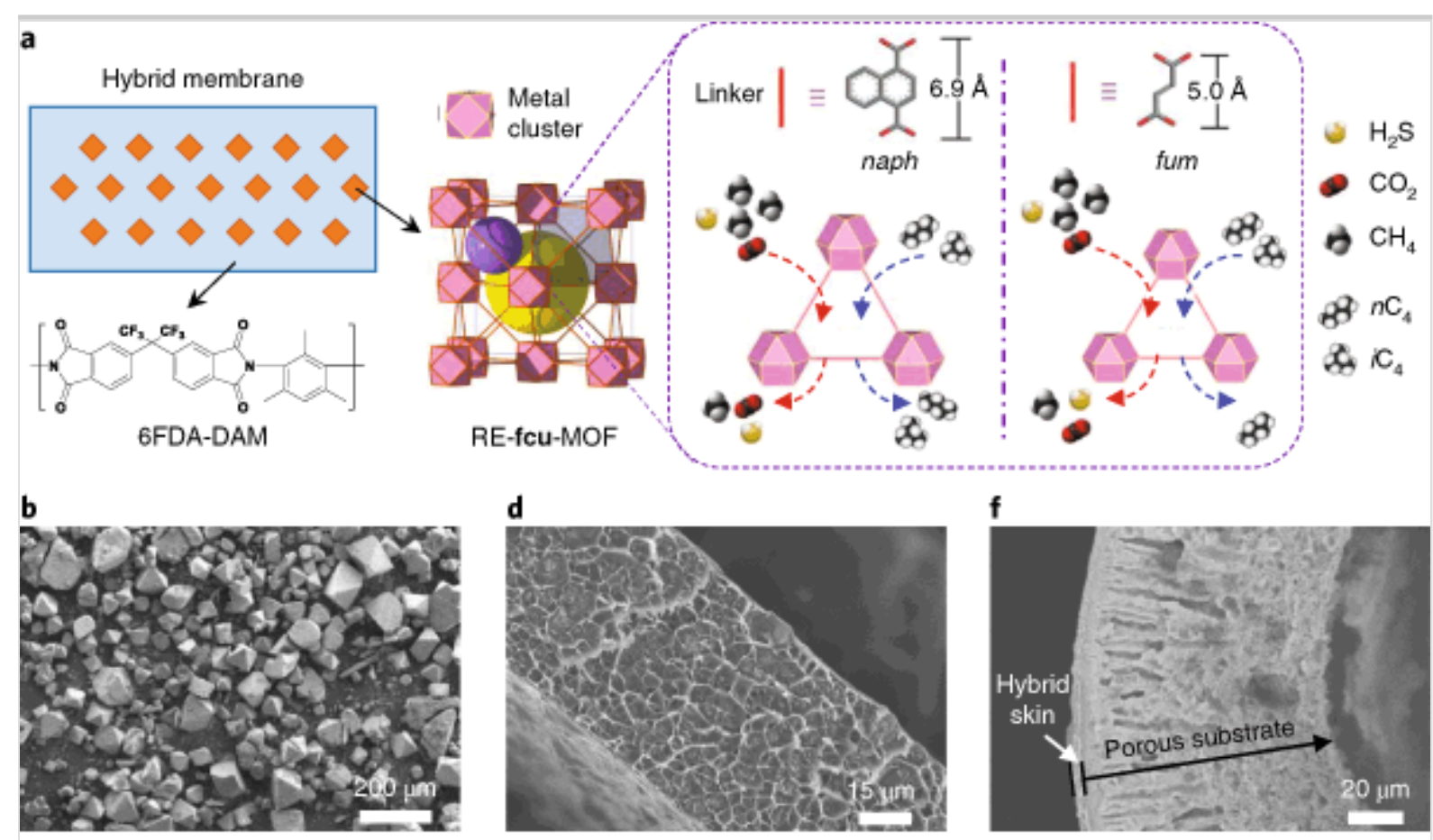

c

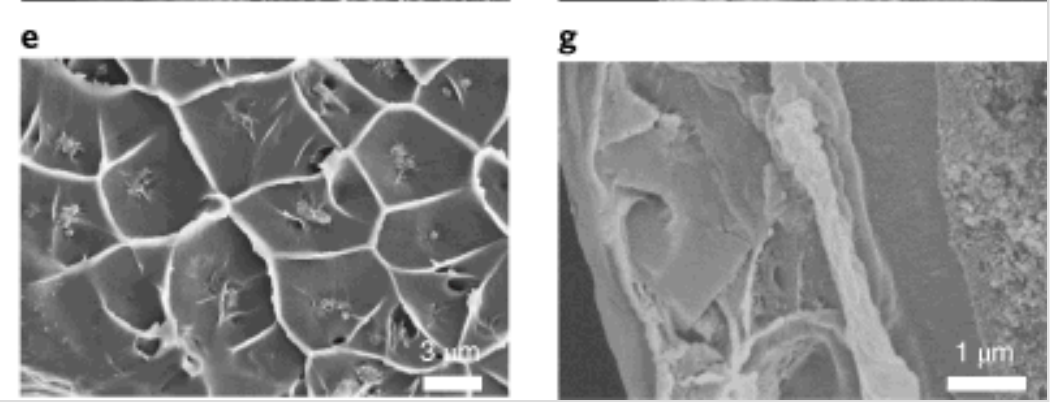




\section{Submicrometre-sized MOFs and mixed-matrix membranes}

Submicrometre-sized particles are ideal for forming ultimately thin-skinned asymmetric membranes for industrial applications[19, 38]. Previous attempts to directly synthesize nano-sized RE-fcu-MOFs molecular sieves had consistently led to the production of large crystals with particle sizes up to hundreds of micrometres (Fig. 1b). Accordingly, we opted to pursue a facile and scalable approach based on cryogenic-grinding/sedimentation of the resultant crystals to produce submicrometre-sized MOF crystals (Supplementary Fig. 1). In contrast to conventional grinding methods at room temperature (Supplementary Fig. 2), a mild manual grinding is carried out at ultralow temperature (for example, $-196{ }^{\circ} \mathrm{C}$ using liquid nitrogen). This straightforward approach allows the structure of the MOFs to be maintained, while making the MOF crystals brittle enough to allow the disassembly of aggregates and weak interfaces/spots in the large crystals (Supplementary Fig. 3). Subsequently, sedimentation can be performed to further purify the ground MOF crystals by dispersing them in a solvent, in which a small fraction of larger micrometre-sized crystals settles, while the majority of submicrometre-sized crystals remain suspended. The sedimentation process can be repeated several times, with the larger settled MOF crystals being further processed in a subsequent cryogenic-grinding/sedimentation cycle. This facile and economical method can be easily scaled up (for example, by simply modifying existing grinding systems), showing potential for the commercial as well as lab-scale fabrication of MOF-based membranes.

The above method efficiently converted as-synthesized Y-fum-fcu-MOF crystals with a wide range of particle size $(10-150 \mu \mathrm{m})$ into relatively uniform submicrometre crystals of size 100-400 nm (Fig. 1b,c). The associated physicochemical properties of these refined crystals were maintained well, as confirmed by X-ray diffraction (XRD), infrared (IR) and gas adsorption measurements (Supplementary Figs. 4-6). These submicrometre-sized Y-fum-fcu-MOF crystalline fillers were then incorporated into a high-performance 6FDA-DAM polyimide to form hybrid membranes. A solution-casting method was used to prepare dense films to study the intrinsic properties of the MOF/polymer hybrid material. As shown in Fig. 1d,e and Supplementary Figs. 7,8, the MOF crystals were uniformly dispersed in the polymer matrix with the requisite excellent MOF/polymer interface.[39] With easy access to submicrometre-sized MOFs, industrially preferred asymmetric hollow fibre membranes[24] could be fabricated via dip-coating. As a first proof of concept, a thin hybrid skin layer with $\sim 5 \mu \mathrm{m}$ thickness was firmly deposited on a highly porous hollow fibre substrate (Fig. 1f,g and Supplementary Figs. 9,10). Similarly, an array of hybrid membranes using different polymer matrices 
(6FDA-DAM-DABA, PEBAX) and incorporating submicrometre-sized isoreticular Eu-naph-fcu-MOF (Supplementary Fig. 11) were prepared and characterized. The studies corroborate the utility of the uniform dispersion of the MOF and the integration of its associated intrinsic properties in the resultant thin film analogous to Y-fum-fcu-MOF/6FDA-DAM (Supplementary Figs. 12-14). XRD, IR and thermogravimetric analysis (TGA) analysis indicated the robust and wellmaintained physicochemical properties of the RE-fcu-MOFs in the hybrid membranes (Supplementary Figs. 15-17).

\section{Pure-gas permeation properties}

Permeation of challenging gas mixtures, $n \mathrm{C}_{4} / i \mathrm{C}_{4}$ and $\mathrm{H}_{2} \mathrm{~S} / \mathrm{CO}_{2} / \mathrm{CH}_{4}$, were performed on RE-fcu-MOF/6FDA-DAM hybrid membranes (Fig. 2). The permeabilities of target molecules $\left(n \mathrm{C}_{4}, \mathrm{CO}_{2}\right.$ and $\left.\mathrm{H}_{2} \mathrm{~S}\right)$ and the selectivities $\left(n \mathrm{C}_{4} / i \mathrm{C}_{4}, \mathrm{H}_{2} \mathrm{~S} / \mathrm{CH}_{4}\right.$ and $\mathrm{CO}_{2} / \mathrm{CH}_{4}$ ) of 6 FDA-DAM membranes are greatly improved by the incorporation of Y-fum-fcu-MOF. This study demonstrates the use of MOF-based membranes for the separation of butane isomers and-and the separation of $\mathrm{H}_{2} \mathrm{~S}$ and $\mathrm{CH}_{4}$ using a particular polymer-MOF pair. A considerably higher $n \mathrm{C}_{4} / i \mathrm{C}_{4}$ selectivity is achieved beyond the current performance limit in polymer and zeolite/polymer hybrid membranes[6, 40,41]. Moreover, the separation performance of the Y-fum-fcu-MOF/6DA-DAM hybrid membranes is systematically enhanced as a function of increased MOF loading up to $30 \mathrm{wt} \%$. The measured permeability and selectivity are consistent with the theoretical framework for mixed-matrix membranes, allowing back-calculation of the permeation properties of the pure Y-fum-fcu-MOF phase (Supplementary Scheme 1)[2]. As expected, the Y-fum-fcu-MOF exhibits a much higher separation performance than the polymer (for example, 6FDA-DAM), providing an effective molecular sieve membrane material for treating natural gas containing $\mathrm{H}_{2} \mathrm{~S}$ and the separation of butane isomers.

\section{Fig. 2}

Gas permeation properties.

a, $n \mathrm{C}_{4}$ permeability and $n \mathrm{C}_{4} / i \mathrm{C}_{4}$ selectivity of Y-fum-fcu-MOF and Eu-naph-fcu-MOF-incorporated 6FDA-DAM hybrid membranes with various MOF loading by wt\%. Pure gas permeations were conducted under 1.72 bar at $75^{\circ} \mathrm{C}$. Other reported polymeric and hybrid membranes are compared. The black line is a guide to the eye and is used only to indicate a general 'trade-off' between permeability and selectivity in reported polymer-based membranes. b, $\mathrm{CO}_{2}$ andor $\mathrm{H}_{2} \mathrm{~S}$ permeability (xaxis), and $\mathrm{CO}_{2} / \mathrm{CH}_{4}$ andor $\mathrm{H}_{2} \mathrm{~S} / \mathrm{CH}_{4}$ selectivity (y-axis) of Y-fum-fcu-MOF/polymer hybrid membranes-with various $\mathrm{MOF}$ 
toadings by $w$ t $\%$ (blue symbols from left to right- represent MOF loadings in 6FDA-DAM $\div$ of 10.6, 17.8, 30.1, and $40 \mathrm{wt} \%$; in 6FDA-DAM-DABA $\div$ of 9.9, 17.7, zo-30; and $40 \mathrm{wt} \%$; in PEBAX $\div$ of $10.1,20.0,30$; and $40 \mathrm{wt} \%$, respectively). To obtain individual permeabilities in model natural gas permeation $\left(20 / 20 / 60 \mathrm{H}_{2} \mathrm{~S} / \mathrm{CO}_{2} / \mathrm{CH}_{4}\right.$ mixtures with total pressure of 6.9 bar), pure $\mathrm{H}_{2} \mathrm{~S}$ or $\mathrm{CO}_{2}$ permeation was conducted under 1.38 bar, and pure $\mathrm{CH}_{4}$ permeation was conducted under 4.14 bar at 35 ${ }^{\circ} \mathrm{C}$. Permeation properties of the pure Y-fum-fcu-MOFs and Y-fum-fcu-MOF/polymer hybrid membranes are predicted by using the measured permeability and selectivity of the 6FDA-DAM membranes and Y-fum-fcu-MOF/6FDA-DAM hybrid membranes according to the theoretical model for mixed-matrix hybrid membranes (see Supplemental Materials Scheme 1). 1 barrer $=10^{-10} \mathrm{cc}(\mathrm{STP}) \mathrm{cm} \mathrm{cm}^{-2} \mathrm{~s}^{-1} \mathrm{cmHg}^{-1}$.

AQ7
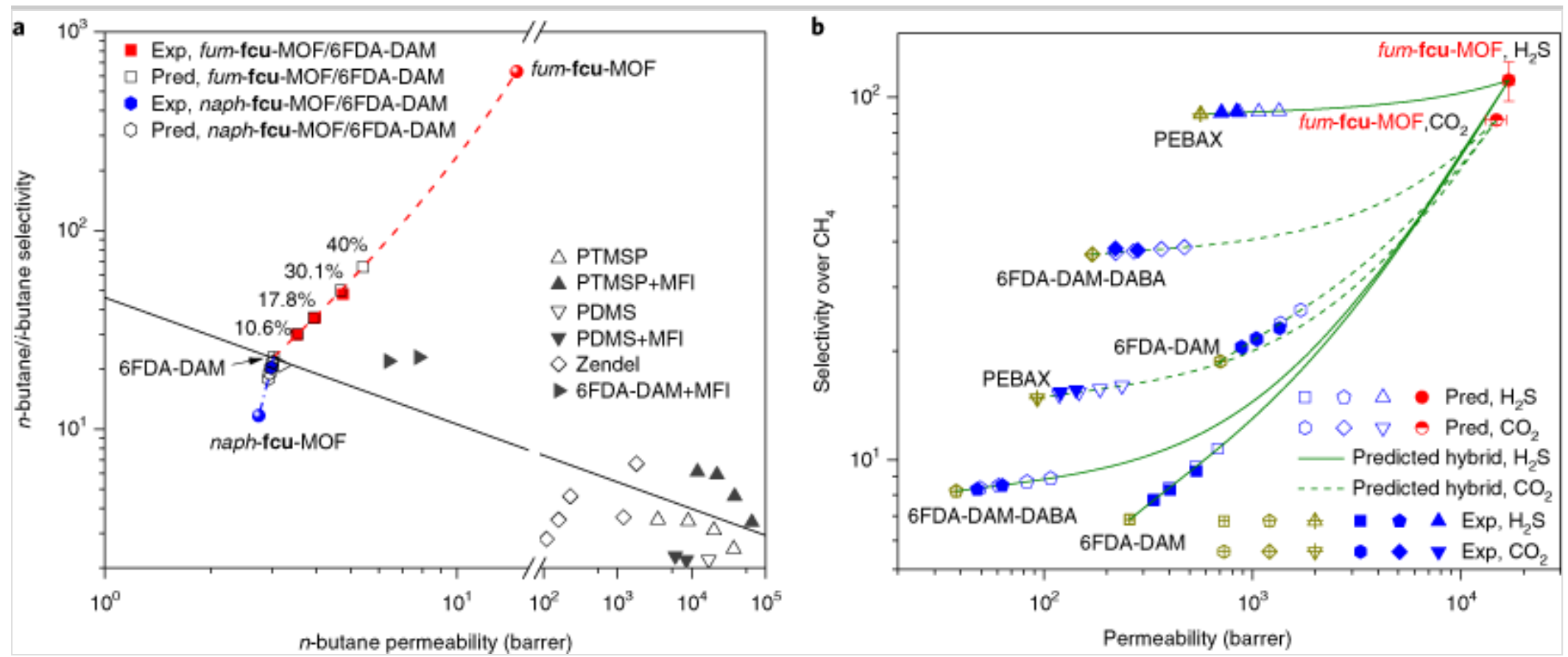

AQ6 
Encouraged by the aforementioned exceptional molecular sieve membrane properties, we further explored the performance of Y-fum-fcu-MOF hybrid membranes using other polymer matrices, including the glassy polymer 6FDA-DAM-DABA[42] and the rubbery polymer PEBAX[43]. Both polymers are known to provide promising properties for $\mathrm{H}_{2} \mathrm{~S} / \mathrm{CO}_{2} / \mathrm{CH}_{4}$ separation. Predictably, both polymeric membranes afforded significantly enhanced performances on introducing the highperformance Y-fum-fcu-MOF sieves. As shown in Fig. 2b, the theoretically predicted permeability and selectivity were successfully validated by the preparation and permeation measurements of various independent Y-fum-fcu-MOF hybrid membranes consisting of the two polymer matrices with two distinct MOF loadings. The Y-fum-fcu-MOF offers excellent interphase compatibility with various polymer matrices, a requisite for the realization of high-performance hybrid mixedmatrix membranes. The excellent agreement between the theoretical predictions and the experimental values demonstrates the mixed-matrix hybrid approach as a simple and powerful tool to determine reliable permeation properties of a pure MOF that are often difficult to determine directly, due to persistent challenges in the fabrication of defect-free pure MOF membranes[30, 44].

The ability to integrate and translate the intrinsic properties of the Y-fum-fcu-MOF, namely the sieving of butane isomers, into a hybrid MOF/polymer membrane motivated us to explore the general practicality of this approach for other members of the RE-fcu-MOF platform - isoreticular RE-fcu-MOFs. Specifically, the Eu-naph-fcu-MOF, incorporating the naphthalene dicarboxylate linker, a relatively bulkier and slightly longer linker than the fumarate in the Y-fum-fcu-MOF, was used to form mixed matrix membranes. As we expected, the hybrid membrane comprising the Eu-naph-fcu-MOF nanoparticles and 6FDA-DAM polymer did not promote the separation of butane isomers, as both butane isomers can freely enter the pore system of the Eu-naph-fcu-MOF through the relatively open triangular window. This lack of control is in strong contrast to the contracted pore-aperture in the Y-fum-fcu-MOF, which was able to discriminate between isobutane and $n$-butane (Fig. 2a). These findings provide further evidence that the exceptional separating properties of the hybrid membrane based on the Y-fum-fcu-MOF were due to the excellent sieving properties of the incorporated Y-fum-fcuMOF. Predictably, enhanced $\mathrm{H}_{2} \mathrm{~S} / \mathrm{CO}_{2} / \mathrm{CH}_{4}$ separation performance was observed in 6FDA-DAM hybrid membranes filled by the Eu-naph-fcu-MOF with various loadings, despite having a lower permeability than the Y-fum-fcu-MOF hybrid membranes (Supplementary Figs. 18,19). The observed molecular sieving performance of RE-fcu-MOFs in the membrane is in accordance with that obtained in the adsorbent form[27, 29,37], illustrating the successful transplantation of the molecular sieving properties of the MOF adsorbent into a hybrid membrane. 


\section{Sorption-diffusion properties and MOFs' pore structure}

To further gain insights into the transport mechanism in RE-fcu-MOF/polymer hybrid membranes, the permeability $\left(P_{i}\right)$ was deconvolved into the product of the sorption coefficient (solubility, $S_{i}$ ) and the diffusion coefficient (diffusivity, $D_{i}$ ), according to the solution-diffusion model[45]. High-pressure sorption measurements ( $0-14$ bar) enabled us to determine the solubility and diffusivity under conditions identical to those needed for the membrane separation process. Sorption isotherms of RE-fcu-MOF crystals, polymer membranes and their hybrid membranes are shown in Supplementary Figs. 20-23. Calculated sorption and diffusion data are summarized in Fig. 3a-c and Supplementary Fig. 24. The sorption of $\mathrm{H}_{2} \mathrm{~S} / \mathrm{CO}_{2} / \mathrm{CH}_{4}$ or $n \mathrm{C}_{4} / i \mathrm{C}_{4}$ in the glassy polymer membrane (6FDA-DAM or 6FDA-DAM-DABA) was not enhanced by incorporating RE-fcu-MOF crystals, which is unusual for MOF-filled mixed-matrix membranes[25]. This presumably reflects the moderate sorption capacity and selectivity of the RE-fcu-MOFs compared with glassy polymers of high freevolume, which is confirmed by sorption measurements of the pure MOF crystals. On the other hand, diffusivity and diffusion selectivity were enhanced in the hybrid membranes for most gases. The variation of transport properties with the addition of RE-fcu-MOFs is also affected by the molecular structural difference in various polymer matrices. In contrast to the glassy polymers, gas diffusion was inhibited in the rubbery PEBAX membrane, probably due to the dispersed MOF crystals reducing the mobility of flexible segments[46]; whereas the low sorption capacity of PEBAX was offset by incorporating the highly porous Y-fum-fcu-MOF. Overall, the greatly improved perm-selectivity either in glassy or rubbery membranes shown in Fig. 2 is attributed to the RE-fcu-MOF sieves providing enhanced selective molecular diffusion-in other words, a molecular sieving property.

\section{Fig. 3}

Transport properties and RE-fcu-MOFs pore structure.

a-c, Solubility (a) and diffusivity (b) of $\mathrm{CO}_{2}, \mathrm{H}_{2} \mathrm{~S}, \mathrm{CH}_{4}, n \mathrm{C}_{4}$ and $i \mathrm{C}_{4}$, and sorption and diffusion selectivity (c) of $\mathrm{CO}_{2} / \mathrm{CH}_{4}$, $\mathrm{H}_{2} \mathrm{~S} / \mathrm{CH}_{4}, n \mathrm{C}_{4} / i \mathrm{C}_{4}$ in 6FDA-DAM, $18 \mathrm{wt} \%$ Y-fum-fcu-MOF/6FDA-DAM and $19 \mathrm{wt} \%$ Eu-naph-fcu-MOF/6FDA-DAM membranes. d, Diffusion coefficients of gases with various molecular diameters in the Y-fum-fcu-MOF. MOF permeability predicted by the mixed-matrix hybrid membranes and solubility obtained from the measured sorption isotherm were used to calculate the gas diffusivity in the Y-fum-fcu-MOF under the same temperature and pressure as used for gas permeation. The 
diffusivities of $n \mathrm{C}_{4}$ and $i \mathrm{C}_{4}$ were also estimated from the sorption kinetics of Y-fum-fcu-MOF crystals. e, Triangular window of RE-fcu-MOFs for gas diffusion. In the Y-fum-fcu-MOF, the linear and rigid fum linker is constrained to a fixed aperture size of $\sim 4.7 \AA$. In the Eu-naph-fcu-MOF, the bulky naph linker with its rotational naphthalene group provides a flexible aperture with a maximum opening size of $\sim 5.0 \AA$. 

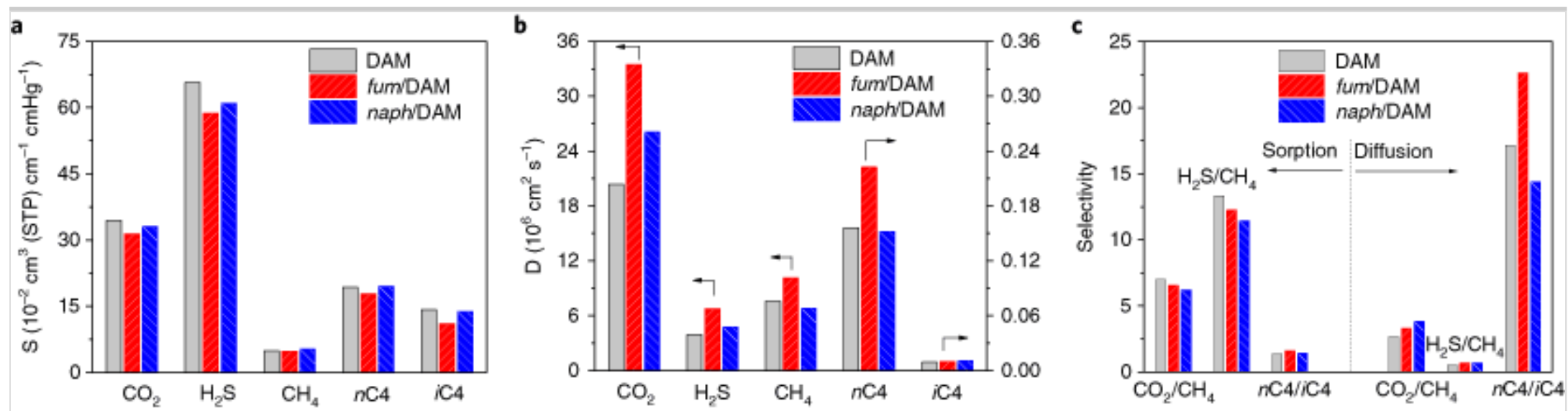

d

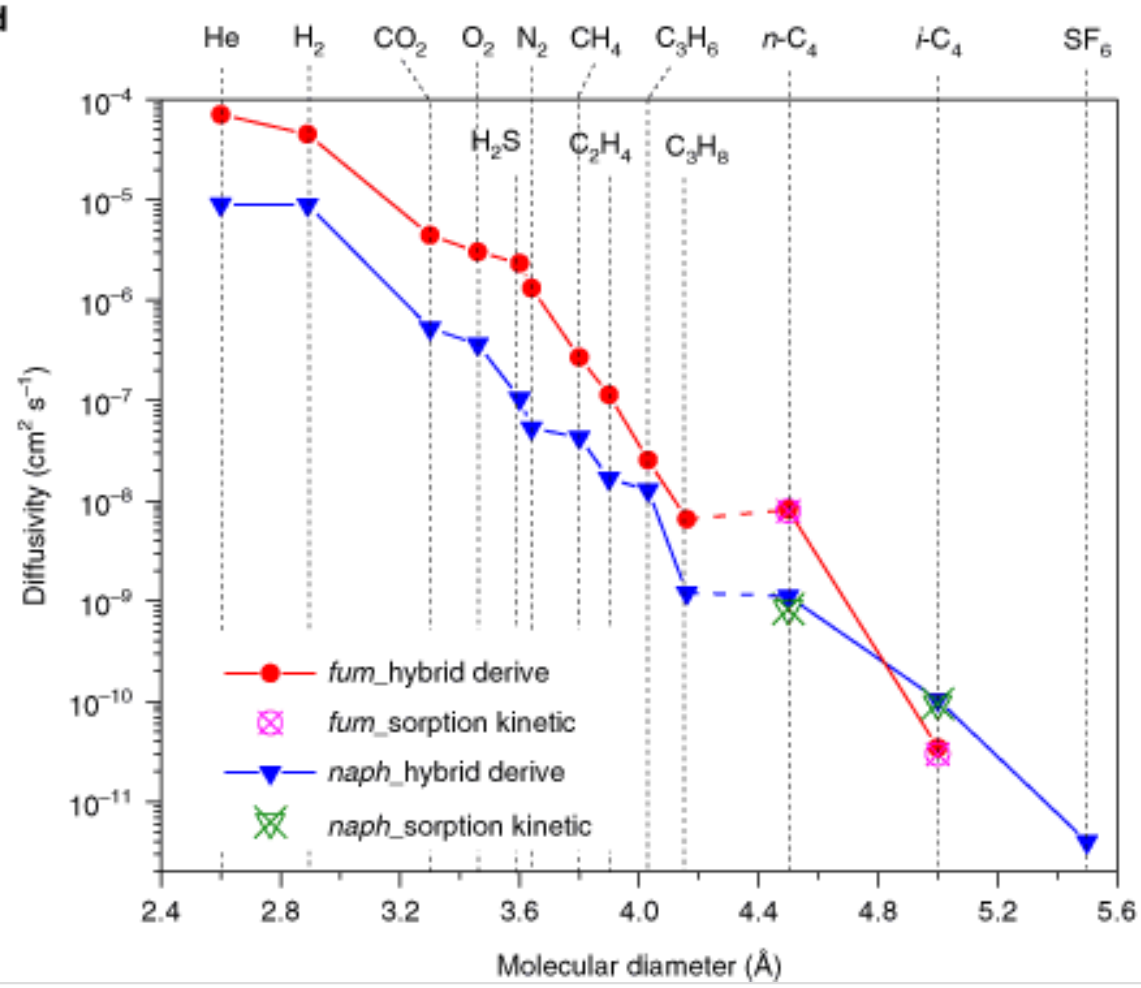

e
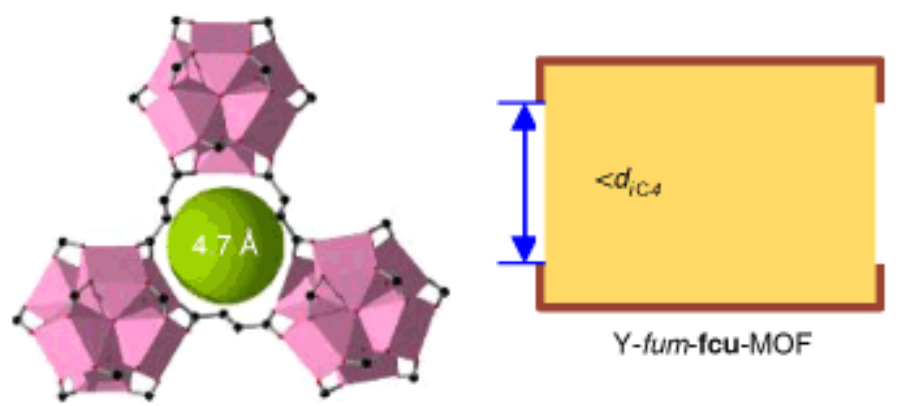

Y-fum-fcu-MOF
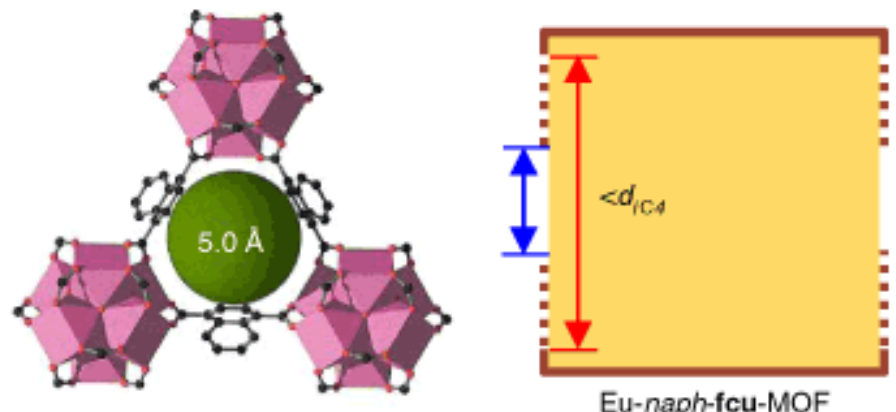

The molecular sieving properties can be finely tuned by varying the ultra-microporous structures of RE-fcu-MOFs using different organic linkers. The naph linker is, of course, longer than the fum linker (length of fum: $5.0 \AA$, naph: 6.9 $\AA$ ) [29], 
offering modular pore systems and tunable sorption capacities in the crystals and the associated hybrid membranes (Fig. 3a). Moreover, the pore-aperture size and associated pore system structure dominate the gas diffusion in RE-fcuMOFs, which can thus be precisely probed by using different sized gases $\left(\mathrm{He}, \mathrm{H}_{2}, \mathrm{CO}_{2}, \mathrm{O}_{2}, \mathrm{H}_{2} \mathrm{~S}, \mathrm{~N}_{2}, \mathrm{CH}_{4}, \mathrm{C}_{2} \mathrm{H}_{4}, \mathrm{C}_{3} \mathrm{H}_{6}\right.$, $\mathrm{C}_{3} \mathrm{H}_{8}, n \mathrm{C}_{4}, i \mathrm{C}_{4}, \mathrm{SF}_{6}$ )[47]. In more constrained slit-shaped pores, the effective molecular diameters can differ slightly from such non-slit pores considered here[48]. The gas diffusivity in MOF can be measured by the permeation of 'pure' MOF membrane or sorption kinetics. Practically, it is challenging to fabricate defect-free pure MOF membranes or very large uniform MOF crystals for gas sorption kinetics studies. Here, we use the predicted permeability and measured gas solubility of mixed-matrix hybrid membranes (Supplementary Fig. 25) to derive gas diffusivities in RE-fcu-MOF, as given in Fig. 3d. The reliability of this facile approach was verified by the good agreement between the butane diffusivities obtained and the diffusivities estimated from butane sorption kinetics of RE-fcu-MOFs crystals.

As expected, RE-fcu-MOFs exhibit a typical molecular sieving characteristic: gas diffusivity is reduced by six orders of magnitude as the molecular diameter increases from 2.6 to $5.5 \AA$. Generally, the RE-fcu-MOF bridged by the linear fum linker provides one order of magnitude higher diffusivity than the bulky naph linker for molecules smaller than $i \mathrm{C}_{4}$. $\mathrm{A}$ sudden diffusivity decrease between $\mathrm{H}_{2} \mathrm{~S}\left(d_{k}=3.6 \AA\right)$ and $\mathrm{CH}_{4}\left(d_{k}=3.8 \AA\right)$ in the RE-fum-fcu-MOF suggests that the hydrogen atoms on the fum linker begin to hinder the diffusion of such molecules. Several plateaus in the gas diffusivity of the Eu-naph-fcu-MOF suggest variable window aperture sizes may be generated by steric hindrances of the naphthalene group with different degrees of rotation (Supplementary Fig. 26)[37]. Exceptionally, the $i \mathrm{C}_{4}$ diffusivity being hindered in the Y-fum-fcu-MOF than in the Eu-naph-fcu-MOF, afforded a significantly enhanced diffusion selectivity for $n \mathrm{C}_{4} / i \mathrm{C}_{4}$ in the Y-fum-fcu-MOF hybrid membranes (Fig. 3c). This ability is due to the window constructed by the shorter fum linker providing a smaller aperture size $(\sim 4.7 \AA)$ than that of the Eu-naph-fcu-MOF[29]. The constrained window aperture of the Y-fum-fcu-MOF shows a size cut-off for $i \mathrm{C}_{4}$, thereby allowing a high $n \mathrm{C}_{4} / i \mathrm{C}_{4}$ diffusion selectivity $\left(\alpha_{D}=237\right)$, that is 21 times higher than that of the Eu-naph-fcu-MOF, and mostly contributing to the high perm-selectivity of the Y-fum-fcuMOF for butane isomers. For the Eu-naph-fcu-MOF, despite having lower diffusivity, presumably caused by the higher energy barrier to rotate the bulkier naphthalene linker, an ultralow diffusivity was observed up to diameters of gas molecules (for example, $\mathrm{SF}_{6}$ ) exceeding the maximum effective opening aperture size of $\sim 5.0 \AA$.

\section{Mixed-gas permeation and performance comparison}


The excellent separation performance of RE-fcu-MOF hybrid membranes was further demonstrated in mixed-gas permeation measurements (Fig. 4). For natural gas purification, the acid gas removal performance can be evaluated by measuring the total acid gas permeability $\left[\mathrm{P}\left(\mathrm{CO}_{2}\right)+\mathrm{P}\left(\mathrm{H}_{2} \mathrm{~S}\right)\right]$ and selectivity $\left.\left[\mathrm{P}\left(\mathrm{CO}_{2}\right)+\mathrm{P}\left(\mathrm{H}_{2} \mathrm{~S}\right)\right] / \mathrm{P}\left(\mathrm{CH}_{4}\right)\right][42]$, since both $\mathrm{CO}_{2}$ and $\mathrm{H}_{2} \mathrm{~S}$ must be removed from $\mathrm{CH}_{4}$. The separation performance of polymer matrices (that is, 6FDA-DAM and 6FDA-DAM-DABA) is greatly improved by introducing RE-fcu-MOFs, with loadings up to $30 \mathrm{wt} \%$. Such RE-fcu$\mathrm{MOF} /$ polymer hybrids have properties far above the performance limit of glassy polymers for $\mathrm{H}_{2} \mathrm{~S} / \mathrm{CO}_{2} / \mathrm{CH}_{4}$ separation (Fig. 4a, Supplementary Tables 1,2). Similarly, simultaneous enhancement in permeability and selectivity was achieved in mixed $n \mathrm{C}_{4} / i \mathrm{C}_{4}$ separation by increasing the MOF loading in Y-fum-fcu-MOF/6FDA-DAM hybrid membranes (Fig. 4b), thus confirming the molecular sieving properties imparted.

\section{Fig. 4}

\section{Mixed-gas separation performance.}

a, $\left(\mathrm{CO}_{2}+\mathrm{H}_{2} \mathrm{~S}\right) / \mathrm{CH}_{4}$ mixed-gas separation performance of pure 6FDA-DAM,6FDA-DAM-DABA, Y-fum-fcu-MOF/6FDADAM, Y-fum-fcu-MOF/6FDA-DAM-DABA and Eu-naph-fcu-MOF/6FDA-DAM hybrid membranes, as well as comparison with glassy polymers reported in the literature; the individual permeabilities of $\mathrm{H}_{2} \mathrm{~S}$ and $\mathrm{CO}_{2}$, and selectivities of $\mathrm{H}_{2} \mathrm{~S} / \mathrm{CH}_{4}$ and $\mathrm{CO}_{2} / \mathrm{CH}_{4}$ are listed in Supplementary Table 1; feed conditions for our membranes: $20 / 20 / 60 \mathrm{H}_{2} \mathrm{~S} / \mathrm{CO}_{2} / \mathrm{CH}_{4}, 6.7$ bar, $35^{\circ} \mathrm{C}$; feed conditions for the reported membranes are provided in Supplementary Table 2; the solid black line is a guide to the eye used only to indicate the general 'trade-off' between permeability and selectivity in glassy polymers; the dashed green and blue lines are guides to the eye used only to indicate the hybrid membranes with RE-fcu-MOF loadings of $\sim 10 \mathrm{wt} \%$ and $\sim 18$ $\mathrm{wt} \%$, respectively; the performance of $18 \mathrm{wt} \%$ Y-fum-fcu-MOF/6FDA-DAM membrane at 55 bar and $35^{\circ} \mathrm{C}$ for $20 / 20 / 60$ $\mathrm{H}_{2} \mathrm{~S} / \mathrm{CO}_{2} / \mathrm{CH}_{4}$ is also included. b, Butane isomers mixed-gas separation performance of Y-fum-fcu-MOF/6FDA-DAM hybrid membranes with various MOF loadings (feed conditions: $50 / 50 n \mathrm{C} 4 / i \mathrm{C} 4,1.72 \mathrm{bar}, 75^{\circ} \mathrm{C}$ ). c, Permeability and selectivity of the $18 \mathrm{wt} \%$ Y-fum-fcu-MOF/6FDA-DAM hybrid membrane for the separation of $20 / 20 / 60 \mathrm{H}_{2} \mathrm{~S} / \mathrm{CO}_{2} / \mathrm{CH}_{4}$ mixtures at $35^{\circ} \mathrm{C}$ under feed pressures ranging from 7 to 55 bar. 

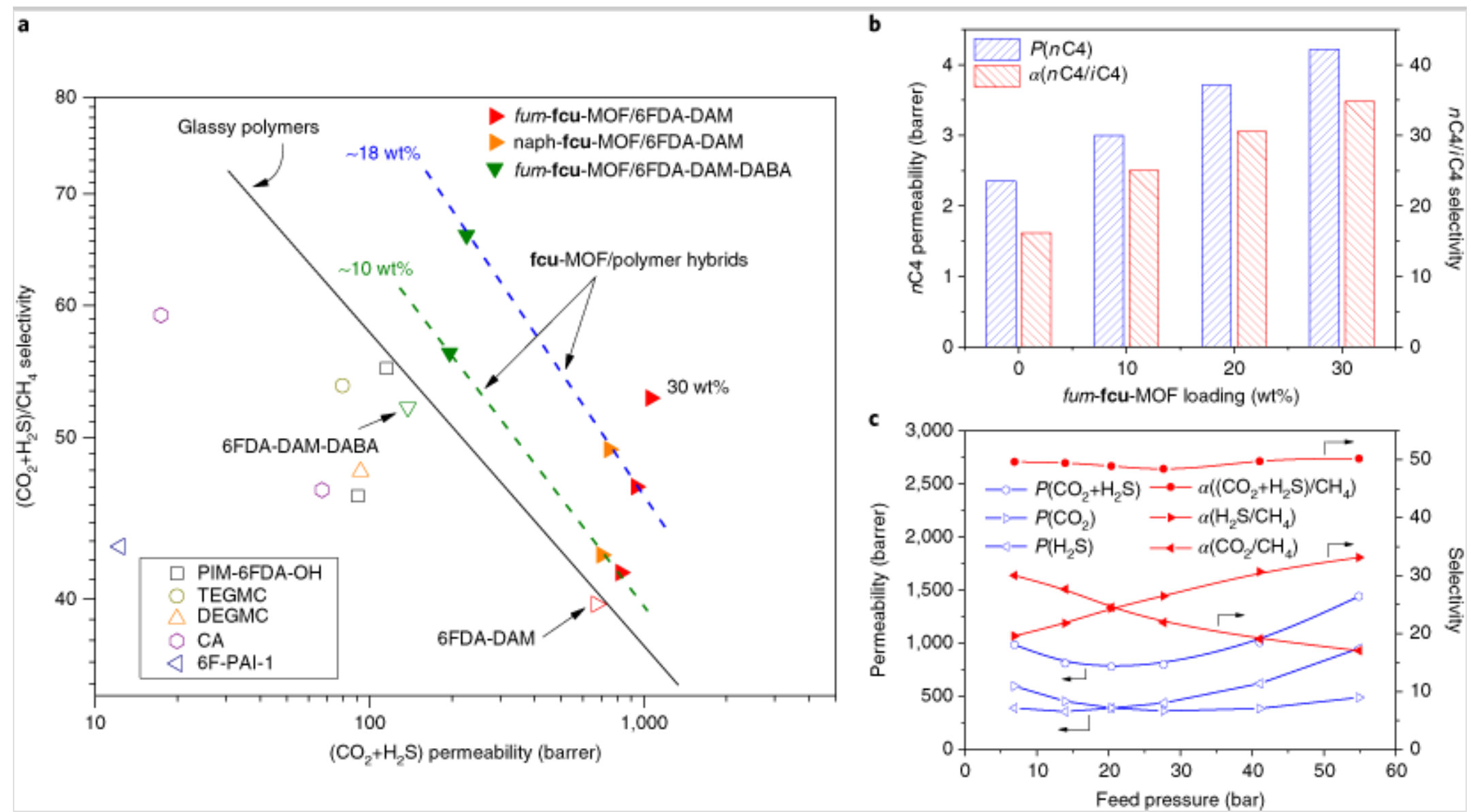

We also evaluated the separation performance of hybrid membranes under aggressive feed conditions that reflect practical natural gas purification[3]. Membrane permeation was studied under high feed pressures up to 55 bar, along with high concentrations of $\mathrm{H}_{2} \mathrm{~S}(20 \%)$ and $\mathrm{CO}_{2}(20 \%)$, As shown in Fig. 4c, the $\mathrm{CO}_{2}$ and $\mathrm{H}_{2} \mathrm{~S}$ permeabilities decrease and then increase with increasing feed pressure, hypothetically due to the pressure-dependent gas solubility and diffusivity in the membrane. Lower solubility is observed in both the 6FDA-DAM and Y-fum-fcu-MOF with increasing feed pressure, which causes the reduction of permeability at low pressures. On the other hand, the increased sorption amounts of condensable $\mathrm{CO}_{2}$ and $\mathrm{H}_{2} \mathrm{~S}$ gases at higher pressures (Supplementary Fig. 22) leads to swelling of the polymer matrix, which enhances the diffusivity and promotes the observed higher permeability. It should be noted that the selectivity is often compromised 
in pure polymeric membranes due to the excessive swelling-induced plasticization[49]. Fortunately, such an undesirable selectivity loss did not occur in the Y-fum-fcu-MOF hybrid membrane for the total acidic gases removal even under 55 bar total pressure and $40 \%$ acid gas containing $20 \% \mathrm{H}_{2} \mathrm{~S}$ or pure $\mathrm{H}_{2} \mathrm{~S}$ up to 12 bar.

The observed stability reflects well-adhered MOF particles within the polymer matrix[25] for operating times exceeding $100 \mathrm{~h}$ (Supplementary Figs. 27). XRD and IR analysis confirmed the intact crystalline and chemical structures of the MOFs and the MOFs hybrid membranes after the high-pressure and long-term sorption and permeation measurements (Supplementary Figs. 5,6). At present, we have commenced the process of translating the RE-fcu-MOF/polymer hybrid dense film into a composite asymmetric hollow fibre membrane of the type that is preferred in industrial implementations. Preliminary results of this effort are reported here. A $13 \mathrm{wt} \% \mathrm{Y}-f u m-\mathbf{f c u}-\mathrm{MOF} / 6 \mathrm{FDA}-\mathrm{DAM}$ hybrid hollow fibre membrane was applied for the separation of $20 / 20 / 60 \mathrm{H}_{2} \mathrm{~S} / \mathrm{CO}_{2} / \mathrm{CH}_{4}$ mixtures at 6.7 bar at $35^{\circ} \mathrm{C}$. Promisingly, the resultant composite fibre exhibited a selectivity for $\left(\mathrm{CO}_{2}+\mathrm{H}_{2} \mathrm{~S}\right) / \mathrm{CH}_{4}$ of 43.9 , in excellent agreement with the results obtained in the dense film (44.2) with a high permeance of $95 \mathrm{GPU}\left(1 \mathrm{GPU}=10^{-6} \mathrm{cc}(\mathrm{STP}) \mathrm{cm}^{-2} \mathrm{~s}^{-1} \mathrm{cmHg}^{-1}\right.$ ) for total acid gases. Such performance is very competitive compared with state-of-the-art membranes for natural gas purification[50], and a more extensive characterization of these promising fibres is underway.

In addition, we studied the separation performance of fcu-MOFs-based membranes for other gas pairs, including $\mathrm{H}_{2} / \mathrm{CH}_{4}$, $\mathrm{CO}_{2} / \mathrm{N}_{2}, \mathrm{CO}_{2} / \mathrm{CH}_{4}$ and $\mathrm{N}_{2} / \mathrm{CH}_{4}$, as well as a comparison with the literature (2008 Robeson upper-bounds[51, 52]) in Supplementary Fig. 28. As expected, the pure fcu-MOFs exhibit excellent permeability and selectivity for these gas pairs, far beyond the upper-bounds for polymeric membranes, except the Eu-naph-fcu-MOF for $\mathrm{N}_{2} / \mathrm{CH}_{4}$. Thus, incorporating these fcu-MOFs could greatly enhance the permeation properties of pure 6FDA-DAM membranes, and the performance gradually increases with fcu-MOF loading. The performance of fcu-MOF/6FDA-DAM membranes, even with MOF loadings as high as $40 \mathrm{wt} \%$, did not surpass the upper-bound for $\mathrm{CO}_{2} / \mathrm{N}_{2}$ or $\mathrm{N}_{2} / \mathrm{CH}_{4}$, owing to the moderate performance of the pure 6FDA-DAM phase for these gas pairs. It can be expected that choosing polymers whose performances are located on the upper-bound to better match the transport properties of the fcu-MOFs could further push the performance of the fcuMOFs hybrid membranes beyond the upper-bound.

\section{Outlook}


Rational incorporation of RE-fcu-MOFs in properly selected polymers can improve available hybrid membrane properties far beyond performance limits for current economically processable materials. Diverse applications beyond the removal of acid gas from natural gas and the separation of butane isomers could also be enabled by this hybrid platform. The demonstrated facile and scalable cryogenic-grinding/sedimentation approach to produce submicrometre-sized MOF crystals hybrid membranes could extend the scope for applications of many MOF crystals. Finally, besides its practical importance, the mixed-matrix hybrid strategy provides a widely applicable fundamental tool for facile characterization of molecular transport in MOFs without requiring the creation of pure component defect-free membranes from such MOFs.

\section{Methods}

\section{Small-scale synthesis of RE-fcu-MOF crystals}

For the Y-fum-fcu-MOF: fumaric acid (10.1 mg, $0.087 \mathrm{mmol}), \mathrm{Y}\left(\mathrm{NO}_{3}\right)_{3} \cdot 6 \mathrm{H}_{2} \mathrm{O}$ (Y = yittrium, $\left.33.4 \mathrm{mg}, 0.087 \mathrm{mmol}\right), 2-$ fluorobenzoic acid (195.0 mg, $1.392 \mathrm{mmol})$ dimethylformamide (DMF) $(2.7 \mathrm{ml})$, and deionized $\mathrm{H}_{2} \mathrm{O}(0.7 \mathrm{ml})$, were combined in a $20 \mathrm{ml}$ scintillation vial, sealed and heated to $115^{\circ} \mathrm{C}$ for $72 \mathrm{~h}$ and cooled to room temperature. The solid was then dried at $150^{\circ} \mathrm{C}$ under vacuum for $24 \mathrm{~h}$ to yield the activated sample. For the Eu-naph-fcu-MOF: $1,4-\mathrm{H}_{2} \mathrm{NDC}(\mathrm{Eu}=$ europium, $9.41 \mathrm{mg}, 0.0435 \mathrm{mmol}), \mathrm{Eu}\left(\mathrm{NO}_{3}\right)_{3} \cdot 5 \mathrm{H}_{2} \mathrm{O}(18.6 \mathrm{mg}, 0.0435 \mathrm{mmol})$, 2-fluorobenzoic acid (48.7 mg, $0.348 \mathrm{mmol}$ ), DMF $(2.2 \mathrm{ml}), \mathrm{H}_{2} \mathrm{O}(0.5 \mathrm{ml})$, and $\mathrm{HNO}_{3}(0.3 \mathrm{ml}, 3.5 \mathrm{M}$ in DMF) were combined in a 20 ml scintillation vial, sealed, and heated to $115^{\circ} \mathrm{C}$ for $60 \mathrm{~h}$, and then cooled to room temperature. The polyhedral crystals were collected and air dried. Assynthesized samples of RE-fcu-MOFs were washed with $10 \mathrm{ml}$ of anhydrous DMF and immersed in $10 \mathrm{ml}$ of ethanol for seven days, during which time the ethanol was replaced three times per day.

\section{Large-scale synthesis of Y-fcu-MOF crystals}

In a typical procedure, a reaction mixture containing $\mathrm{Y}\left(\mathrm{NO}_{3}\right)_{3} \cdot 6 \mathrm{H}_{2} \mathrm{O}(0.0105 \mathrm{~mol}, 4.02 \mathrm{~g})$, fumaric acid (0.0105 mol, 1.22 g), 2-fluorobenzoic acid ( $0.105 \mathrm{~mol}, 14.71 \mathrm{~g})$, water $(15 \mathrm{ml})$ and DMF $(125 \mathrm{ml})$ was added to a $250 \mathrm{ml}$ pressure flask, then the flask was placed in an oil bath and heated with stirring to $150^{\circ} \mathrm{C}$ for two days. The solution was then cooled to room temperature. The white precipitate (fcu-MOF) was washed several times with DMF and then exchanged with ethanol for seven days. The obtained yield is $3.2 \mathrm{~g}$. The as-synthesized micrometre-sized Y-fum-fcu-MOF or Eu-naph-fcu-MOF 
crystals were manually ground in a mortar soaked in liquid nitrogen for $20 \mathrm{~min}$. The ground samples were dispersed in dichloromethane (DCM) to form a $10 \mathrm{wt} \%$ suspension. After settling for $5 \mathrm{~min}$, the submicrometre-sized MOF crystals suspended in DCM were pipetted out, and the settled large MOF crystals were dispersed in DCM, which then underwent another 5 min sedimentation process. The settled large MOF crystals were also dried, cryogenically ground and purified by sedimentation in DCM following the above-mentioned procedures. The resulting DCM suspension containing purified submicrometre-sized fcu-MOF crystals was washed with solvent (THF or $n$-butanol) for the mixed-matrix membrane preparation. The MOF content in the suspension was determined by drying a certain weight of MOF/solvent suspension and weighing the dried MOFs.

\section{Synthesis of polymers}

Polyimide polymers were synthesized via condensation of dianhydride monomers with diamine. Typically, stoichiometric amounts of monomers and diamines were agitated and reacted in a $20 \mathrm{wt} \% 1$-methyl-2-pyrrolidinone (NMP) solution under $\mathrm{N}_{2}$ purge at low temperature $\left(\sim 5^{\circ} \mathrm{C}\right)$ for $24 \mathrm{~h}$ to produce a high-molecular-weight PAA polyamide acid solution. Then chemical imidization was achieved in the presence of beta picoline and acetic anhydride at ambient temperature for $24 \mathrm{~h}$ under $\mathrm{N}_{2}$ purge, and the resulting polyimide was precipitated and washed with methanol and dried at $210{ }^{\circ} \mathrm{C}$ under vacuum for $24 \mathrm{~h}$. Monomers comprising (4,4'-hexafluoroisopropylidene) diphthalic anhydride (6FDA) and diamine 2,4,6-trimethyl1,3-diaminobenzene (DAM) were used to synthesize 6FDA-DAM polymer. The molecular weight of the synthesized 6FDA-DAM was $210,000 \mathrm{~g} \mathrm{~mol}^{-1}$ with a polydispersity index (PDI) of 2.55. Also, monomers comprising 6FDA, DAM and 3,5-diaminobenzoic acid (DABA) were used to synthesize 6FDA-DAM-DABA polymer with a DAM/DABA molar ratio of $3: 2$. The molecular weight of the synthesized 6FDA-DAM-DABA was $226,000 \mathrm{~g} \mathrm{~mol}^{-1}$ with a PDI of 2.46. PEBAX is a commercially available polyether-amide block copolymer, and its general chemical structure is shown in Supplementary Scheme 2. PEBAX 1074, which was used in this work, contains $55 \mathrm{wt} \%$ polyoxyethylene (PEO) and $45 \mathrm{wt} \%$ polyamide (PA) 12 (12 repeated units of PA), and was purchased from Arkema Inc. Technical Polymers. AQ8

\section{Membrane preparation}


The mixed matrix membranes were prepared according to previously reported techiques[23]; the procedure is reproduced here for completeness.

Pure polyimide dense films and RE-fcu-MOF/polyimide mixed-matrix dense films

Polyimides (6FDA-DAM or 6FDA-DAM-DABA) were dried in a vacuum oven at $110^{\circ} \mathrm{C}$ overnight before being dissolved in THF to form a $15 \mathrm{wt} \%$ polyimide/THF solution. The solution was mixed on a rolling mixer overnight to dissolve the polymer. The resulting casting solution was poured onto a glass plate, which was placed in a glove bag pre-saturated with THF vapour for at least $4 \mathrm{~h}$. Pure 6FDA-DAM or 6FDA-DAM-DABA dense films were formed on a glass plate by casting the solution to the desired thickness (typically $75 \mu \mathrm{m}$ ) using a draw knife with the appropriate specific clearance. The film was left in the glove bag overnight to allow the THF solvent to evaporate slowly. The film was further dried in a vacuum oven at $200{ }^{\circ} \mathrm{C}$ overnight to remove any remaining solvent. For mixed-matrix dense film formation, the submicrometresized Y-fum-fcu-MOF or Eu-naph-fcu-MOF/THF suspension was added to $10 \mathrm{wt} \%$ polyimide/THF solution to form the mixed-matrix solution, which was mixed on a rolling mixer overnight. Excess solvent in the mixed-matrix solution was removed by slowly purging dry nitrogen to obtain an $\sim 15 \mathrm{wt} \%$ mixed-matrix/THF solution. Y-fum-fcu-MOF/6FDA-DAM, Y-fum-fcu-MOF/6FDA-DAM-DABA or Eu-naph-fcu-MOF/6FDA-DAM mixed matrix dense films were formed by casting the mixed-matrix solution under the same conditions as for the pure 6FDA-DAM and 6FDA-DAM-DABA dense films.

\section{Pure PEBAX dense film and RE-fcu-MOF/PEABX mixed-matrix dense films}

PEBAX was dried in a vacuum oven at $110^{\circ} \mathrm{C}$ overnight before being dissolved in $n$-butanol at $90{ }^{\circ} \mathrm{C}$ for at least $8 \mathrm{~h}$ to form a $15 \mathrm{wt} \% \mathrm{PEBAX} /$ butanol solution. Pure PEBAX dense film was formed by pouring the hot solution into a Teflon dish in a hood. The film was left in the hood overnight to allow the THF solvent to evaporate slowly, and then further dried in a vacuum oven at $70^{\circ} \mathrm{C}$ overnight to remove any remaining solvent. For mixed-matrix dense film formation, the submicrometre-sized Y-fum-fcu-MOF/butanol suspension was added to $10 \mathrm{wt} \% \mathrm{PEBAX} / \mathrm{butanol}$ solution to form a mixedmatrix solution under stirring at $70{ }^{\circ} \mathrm{C}$ for at least $4 \mathrm{~h}$. Excess solvent in the mixed-matrix solution was removed by slowly purging dry nitrogen to obtain an $\sim 15 \mathrm{wt} \%$ mixed-matrix/butanol solution. Y-fum-fcu-MOF/PEBAX mixed matrix dense films were formed by casting the mixed-matrix solution under the same conditions as for the pure PEBAX dense film. MOF loadings in mixed-matrix dense films were controlled by changing the mass ratio of the RE-fcu-MOF to the polymers 
in the mixed-matrix solution. The RE-fcu-MOF loadings in mixed matrix dense films were determined by TGA, as will be described in detail later.

Pure 6FDA-DAM asymmetric hollow fibre membrane and Y-fcu-fcu-MOF/6FDA-DAM asymmetric hollow fibre membrane

6FDA-DAM polymers were dried in a vacuum oven at $110^{\circ} \mathrm{C}$ overnight before being dissolved in THF/DCM (molar ratio $2: 1)$ to form a $3 \mathrm{wt} \% 6 \mathrm{FDA}-\mathrm{DAM} /(\mathrm{THF}+\mathrm{DCM})$ solution. Pure 6FDA-DAM asymmetric hollow fibre membrane was formed by dip-coating the solution on the outer surface of porous 6FDA-BPDA-DAM/(P84 $\left.+\mathrm{SiO}_{2}\right)$ hollow fibre (BPDA: 3,3',4,4'-biphenyl tetracarboxylic dianhydride, P84: a brand name of polyimides manufactured by Evonik). For the mixedmatrix asymmetric hollow fibre membrane, Y-fum-fcu-MOF/DCM solution was mixed with 6FDA-DAM/THF solution to form a $3 \mathrm{wt} \%$ (Y-fum-fcu-MOF + 6FDA-DAM)/(THF + DCM) solution with a THF/DCM molar ratio of 2:1. Y-fum-fcuMOF/6FDA-DAM composite asymmetric hollow fibre membrane was formed by dip-coating the solution on the outer surface of the porous 6FDA-BPDA-DAM/(P84 $\left.+\mathrm{SiO}_{2}\right)$ hollow fibre using the same procedure as for preparing pure 6FDADAM asymmetric hollow fibre membrane.

\section{Membrane characterizations}

The dense films and hollow fibres for the scanning electron microscope (SEM, Hitachi, SU8010) tests were prepared by first soaking films or fibres in hexane and then cryogenically fracturing fibres in liquid nitrogen to preserve their microstructures. Wide-angle X-ray diffraction (WAXD) was measured on a Panalytical Empyrean diffractometer operating with $\mathrm{Cu} \mathrm{K} \alpha$ radiation at a wavelength of $1.54 \AA$, in the $2 \theta$ range $5-45^{\circ}$. Attenuated total reflectance Fourier transform infrared (ATR-FTIR) spectra were recorded by a Thermo Scientific iS50 spectrophotometer at a resolution of $4 \mathrm{~cm}^{-1}$ with 64 scans in the spectral range $4000-400 \mathrm{~cm}^{-1}$. Thermogravimetric analysis (TGA) and derivative weight data were recorded on a TA Q-500 analyzer at a heating rate of $10^{\circ} \mathrm{C} \mathrm{min}^{-1}$ under a nitrogen atmosphere, using an $\sim 10 \mathrm{mg}$ sample. The MOF loading in mixed-matrix dense films was determined by the TGA method. The RE-fcu-MOFs powders were first activated under flowing $\mathrm{N}_{2}$ at $200^{\circ} \mathrm{C}$ for $2 \mathrm{~h}$ to ensure activation, then heated to $800^{\circ} \mathrm{C}$ at a heating rate of $10^{\circ} \mathrm{C} \mathrm{min}^{-1}$ under flowing $\mathrm{O}_{2}$ and held at $800^{\circ} \mathrm{C}$ for $2 \mathrm{~h}$. The remaining oxide mass was compared to the initial activated mass of the MOF. The same protocol was conducted for the RE-fcu-MOF mixed-matrix films. The percentage of mass remaining after $800{ }^{\circ} \mathrm{C}$ for $2 \mathrm{~h}$ under flowing $\mathrm{O}_{2}$ is attributed to metal oxide. Thus, the weight percentage of RE-fcu-MOFs in the mixed- 
matrix film was obtained. Typical TGA curves under $\mathrm{O}_{2}$ atmosphere for determining the RE-fcu-MOF loading in the mixed-matrix hybrid membranes are shown in Supplementary Fig. 29.

\section{Gas permeation measurement}

The gas permeation measurements were performed according to a previously reported prcedure[42], which is reproduced here for completeness. The gas permeation was conducted in a variable-pressure, constant-volume apparatus (Supplementary Scheme 3). The membrane was housed between an upstream volume, capable of introducing high-pressure gas, and a downstream volume, which was kept under vacuum until the experiments were initiated. Further details of the 'constant-volume' permeation have been described elsewhere[42]. Modifications to the standard system configuration were made to ensure safe handling of the toxic $\mathrm{H}_{2} \mathrm{~S}$-containing gases. Specifically, two pneumatically actuated valves were used in place of standard hand-operated valves. These are located between the upstream volume and the membrane-containing cell, and between the downstream volume and the gas chromatograph sampling line, which is normally under vacuum. These valves were controlled by a LabVIEW program that was used for data collection to prevent over-pressurization of the downstream volume, which can damage the downstream pressure transducer, and also to avoid any unintended release of large quantities of $\mathrm{H}_{2} \mathrm{~S}$ gas and to minimize operator risk when handling $\mathrm{H}_{2} \mathrm{~S}$. The permeation of $n$-butane and isobutane was carried out at $75^{\circ} \mathrm{C}$ to avoid possible plasticization of polymer membranes. The permeation temperature for other gases was kept at $35^{\circ} \mathrm{C}$.

A $5 / 95 n$-butane/iso-butane mixture or a $20 / 20 / 60 \mathrm{H}_{2} \mathrm{~S} / \mathrm{CO}_{2} / \mathrm{CH}_{4}$ mixture were used for mixed-gas permeation tests. The downstream composition was determined using a gas chromatograph (Varian 450-GC). The stage cut (the flow rate ratio of permeate to feed) was maintained below $1 \%$ to avoid concentration polarization on the upstream side of the permeation cell and keep the driving force across the membrane constant throughout the course of the experiment. For permeation 20/20/60 $\mathrm{H}_{2} \mathrm{~S} / \mathrm{CO}_{2} / \mathrm{CH}_{4}$ mixtures, a syringe pump and metering valve were used to maintain the feed pressure and control the retentate flow, respectively. The syringe pump is operated isobarically to replenish the feed gas at the same rate as retentate vents through the metering valve.

\section{Gas sorption measurement}


Gas sorption isotherms at feed pressures up to 10 bar were measured using a pressure decay method[5152] (Supplementary Scheme 4). The method and apparatus have been described in detail elsewhere. The gas adsorption amount in RE-fcuMOFs and dense films was calculated from the pressure change before and after sorption. A small experimental error of less than $\pm 5 \%$ is expected from the accurate pressure measurement and volume calibration. The sorption measurements of $n$-butane and iso-butane were conducted at $75^{\circ} \mathrm{C}$, whereas those of other gases were carried out at $35^{\circ} \mathrm{C}$.

Films or MOF particles were activated at $200^{\circ} \mathrm{C}$ under vacuum for $24 \mathrm{~h}$ and then loaded into the cell (B) and degassed for overnight. A differential amount of sorption gas was first introduced into the reservoir (A), then the connecting valve was quickly opened for 1 second and closed. The pressure signal in both volume (A) and volume (B) were recorded continuously. The gas adsorption concentration in films or MOFs was calculated through the molar balance: the adsorbed amount equals the decreased amount in the reservoir (A) minus the increased amount of gas in the cell (B), as below:

$$
C=\frac{\frac{V_{\mathrm{A}}}{R T}\left(\frac{P_{\mathrm{A}, i}}{Z_{\mathrm{A}, i}}-\frac{P_{\mathrm{A}, f}}{Z_{\mathrm{A}, f}}\right)-\frac{V_{\mathrm{B}}}{R T}\left(\frac{P_{\mathrm{B}, f}}{Z_{\mathrm{B}, f}}-\frac{P_{\mathrm{B}, i}}{Z_{\mathrm{B}, i}}\right)}{V_{\text {sample }}}
$$

\section{Calculations of permeability, solubility and diffusivity}

Permeability (or permeance) and selectivity are used to characterize the membrane separation performance. The permeability, $P_{i}$, describes the intrinsic gas separation productivity of a dense film membrane and is defined by the flux of penetrant $i, n_{i}$, normalized by the membrane thickness, $l$, and the partial pressure or fugacity difference, $\Delta p_{i}$, across the membrane, as shown in the following equation:

$$
P_{i}=\frac{n_{i} l}{\Delta p_{i}}
$$

To obtain the permeability from the constant-volume, variable-pressure permeation system, the following quantities can be used: slope of the permeate pressure versus time $(d p / d t)$, membrane thickness $(l)$, downstream volume $(V)$, operating 
temperature $(T)$, and transmembrane pressure or fugacity difference $(\Delta p)$, which can be plugged into equation 2 to obtain the permeability $(P)$ :

$$
P=\frac{\frac{d p}{d t} l V}{A T \Delta p}
$$

In the mixed-gas permeation, the permeability of component $i\left(P_{i}\right)$ is calculated by using its molar fraction in the permeate $\left(x_{i}\right)$ and the transmembrane fugacity difference $\left(\Delta p_{i}\right)$ :

$$
P_{i}=\frac{\frac{d p}{d t} x_{i} l V}{A T \Delta p_{i}}
$$

The fugacity coefficients of $\mathrm{H}_{2} \mathrm{~S}, \mathrm{CO}_{2}, \mathrm{CH}_{4}, n-\mathrm{C}_{4} \mathrm{H}_{10}$ and $i-\mathrm{C}_{4} \mathrm{H}_{10}$ in gas mixtures can be calculated using the PengRobinson equation-of-state and the SUPERTRAPP program developed by NIST.

The permeance of hollow fibre composite membranes is calculated as:

$$
\frac{P}{l}=\frac{\frac{d p}{d t} V}{A T \Delta p}
$$

The membrane selectivity, $\alpha_{i j}$, is determined by the ratio of the permeability or permeance of the fast gas $(i)$ to the slow gas $(j)$ :

$$
\alpha_{i j}=\frac{P_{i}}{P_{j}}=\frac{P_{i} / l}{P_{j} / l}
$$


Permeability can also be expressed as the product of the effective diffusion coefficient $(D)$ and sorption coefficient $(S)$ of a given gas $i$ within the membrane:

$$
P_{i}=D_{i} S_{i}
$$

The sorption coefficient represents the thermodynamic contribution to transport. It can be measured independently by pressure-decay sorption and expressed as:

$$
S_{i}=\frac{c_{i}}{f_{i}}
$$

where $c_{i}$ is the concentration of a gas adsorbed in the sample, and $f_{i}$ is the corresponding upstream fugacity driving force of component $i$.

For glassy polymers (6FDA-DAM, 6FDA-DAM-DABA) and their mixed-matrix films, the adsorbed gas concentration can be described by the dual-mode sorption model, which is given as:

$$
c_{i}=c_{\mathrm{D}, i}+c_{\mathrm{H}, i}=k_{\mathrm{D}, i} f_{i}+\frac{C^{\prime}{ }_{\mathrm{H}, i} b_{i} f_{i}}{1+b_{i} f_{i}}
$$

where $c_{\mathrm{D}, i}$ is the Henry's law or dissolved mode penetrant concentration, $c_{\mathrm{H}, i}$ is the penetrant concentration in the Langmuir mode or hole-filling sorption mode, $k_{\mathrm{D}, i}$ is the Henry's law sorption coefficient, $C_{\mathrm{H}, i}^{\prime}$ is the Langmuir capacity constant, and $b_{i}$ is the Langmuir affinity constant.

For rubbery polymer (PEBAX) and its mixed-matrix films, the adsorbed gas concentration can be described by Henry's law:

$$
c_{i}=c_{\mathrm{D}, i}=k_{\mathrm{D}, i} f_{i}
$$


For RE-fcu-MOF crystals, the adsorbed concentration of $\mathrm{H}_{2}, \mathrm{He}, \mathrm{N}_{2}$ or $\mathrm{O}_{2}$ was also described by the above Henry's law equation, while the adsorbed concentrations of $\mathrm{CO}_{2}, \mathrm{H}_{2} \mathrm{~S}, \mathrm{CH}_{4}, \mathrm{C}_{2} \mathrm{H}_{4}, \mathrm{C}_{2} \mathrm{H}_{6}, \mathrm{C}_{3} \mathrm{H}_{6}, \mathrm{C}_{3} \mathrm{H}_{8}, n-\mathrm{C}_{4} \mathrm{H}_{10}$ or $i$ - $\mathrm{C}_{4} \mathrm{H}_{10}$ were described by the Langmuir model:

$$
c_{i}=c_{\mathrm{H}, i}=\frac{C_{\mathrm{H}, i}^{\prime} b_{i} f_{i}}{1+b_{i} f_{i}}
$$

The effective diffusion coefficient $(D)$ in the membrane or MOF crystals $\left(D_{i}\right)$ can be calculated from the independently measured permeability $(P)$ and sorption coefficient $(S)$ :

$$
D_{i}=\frac{P_{i}}{S_{i}}
$$

The diffusivity $(D)$ of the RE-fcu-MOFs can be calculated by fitting the experimental kinetic uptake curve $\left(M_{t} / M_{\infty} \sim t^{1 / 2}\right)$ obtained from kinetic uptake rate measurements with the theoretical uptake curve derived from an analytical solution to the model of transient diffusion with intracrystalline diffusion control[53]. For a spherical particle subject to a step change in adsorbate concentration at its external surface at time zero, the fractional uptake is equal to[54]:

$$
\frac{M_{t}}{M_{\infty}}=1-\frac{6}{\pi} \sum_{n=1}^{\infty} \frac{1}{n^{2}} \exp \left(-\frac{n^{2} \pi^{2} D t}{R^{2}}\right)
$$

where $M_{t}$ and $M_{\infty}$ are moles adsorbed during time $t$ and as $t \rightarrow \infty, D$ is the transport diffusivity, and $R$ is the equivalent spherical crystal radius of the adsorbent particle. The equation assumes that the percentage change in the external boundary condition was less than $\sim 10 \%$. In our pressure decay method, the absolute pressure in the cell changed by less than $10 \%$ during the sorption run by taking small enough pressure steps, which could meet the assumption. In the short-time region, the kinetic uptake curve is essentially linear and approximated by: 


$$
\frac{M_{t}}{M_{\infty}} \approx \frac{6}{\sqrt{\pi}} \sqrt{\frac{D t}{R^{2}}}
$$

The Maxwell model is used to mathematically describe the gas transport in mixed-matrix materials[2]. The model was initially derived by James C. Maxwell to describe dielectric properties in a conducting dilution suspension of identical particles, and is used to describe gas transport in mixed-matrix materials based on the close analogy between electrical conduction and gas permeation. The Maxwell model is given by:

$$
P_{\mathrm{MMM}}=P_{\mathrm{p}}\left[\frac{P_{\mathrm{s}}+2 P_{\mathrm{p}}-2 \phi_{\mathrm{s}}\left(P_{\mathrm{p}}-P_{\mathrm{s}}\right)}{P_{\mathrm{s}}+2 P_{\mathrm{p}}+\phi_{\mathrm{s}}\left(P_{\mathrm{p}}-P_{\mathrm{s}}\right)}\right]
$$

where $P_{\mathrm{MMM}}$ is the permeability in the mixed matrix material; $P_{\mathrm{p}}$ is the permeability in the polymer matrix; $P_{\mathrm{s}}$ is the permeability in dispersed molecular sieve particles, and $\phi_{\mathrm{s}}$ is the volume fraction of molecular sieve particles in the mixed matrix material.

\section{Data availability}

The data that support the plots within this paper and other findings of this study are available from the corresponding authors upon reasonable request.

\section{Methods}

Methods, including statements of data availability and any associated accession codes and references, are available at https://doi.org/10.1038/s41563-017-0013-1.

Publisher's note: Springer Nature remains neutral with regard to jurisdictional claims in published maps and institutional affiliations.

\section{Supplementary information}


Supplementary information is available for this paper at https://doi.org/10.1038/s41563-017-0013-1.

\section{Acknowledgements}

The research reported in this publication was supported by KAUST CRG Research Grant URF/1/2222-01; Y.B., O.S. and M.E. acknowledge support from King Abdullah University of Science and Technology; G.L. acknowledges support from National Natural Science Foundation of China (Grant Nos.: 21490585, 21776125, 21406107).

\section{Author contributions}

G.L. fabricated the hybrid mixed-matrix membranes and performed the adsorption and permeation tests. V.C., O.S. and Y.B. carried out the synthesis and scale-up of the MOFs. G.L., W.J.K., M.E. and Y.B. interpreted the adsorption and permeation data. K.Z., G.L., O.S. and V.C. developed the cryo-grinding/sedimentation method. K.Z. and G.L. fabricated the hybrid hollow fibre composite membranes. C.Z. guided the Maxwell prediction and diffusivity calculations. S.Y. guided the $\mathrm{H}_{2} \mathrm{~S}$ sorption and permeation. W.J.K. and M.E conceived, designed and guided the whole project. G.L., Y.L., W.J.K., Y.B. and M.E. discussed the findings in this paper. G.L., Y.B. M.E. and W.J.K. coordinated the writing of the paper, and all authors contributed to revising the paper.

Competing interests The authors declare no competing financial interests.

\section{Supplementary information}

\section{Supplementary Information}

Supplementary Figures 1-29, Supplementary Schematic 1-4, Supplementary Tables 1-2, Supplementary References 1-7

\section{References}


1. Koros, W. J. \& Lively, R. P. Water and beyond: Expanding the spectrum of large-scale energy efficient separation processes. AIChE J. 58, 2624-2633 (2012).

2. Koros, W. J. \& Zhang, C. Materials for next-generation molecularly selective synthetic membranes. Nat Mater 16, 289-297 (2017).

3. Baker, R. W. \& Lokhandwala, K. Natural gas processing with membranes: An overview. Ind. Eng. Chem. Res. 47, 2109-2121 (2008).

4. Klemola, K. T. \& Ilme, J. K. Distillation efficiencies of an industrial-scale $i$-butane/ $n$-butane fractionator. Ind. Eng. Chem. Res. 35, 4579-4586 (1996).

5. Baker, R. W. \& Low, B. T. Gas separation membrane materials: A perspective. Macromolecules 47, 6999-7013 (2014).

6. Liu, J. et al. Butane isomer transport properties of 6FDA-DAM and MFI-6FDA-DAM mixed matrix membranes. $J$. Memb. Sci. 343, 157-163 (2009).

7. Agrawal, K. V. et al. Oriented MFI membranes by gel-less secondary growth of sub-100 nm MFI-nanosheet seed layers. Adv. Mater. 27, 3243-3249 (2015).

8. Park, H. B., Kamcev, J., Robeson, L. M., Elimelech, M. \& Freeman, B. D. Maximizing the right stuff: The trade-off between membrane permeability and selectivity. Science 356, eaab0530 (2017).

9. Bae, T.-H. et al. Facile high-yield solvothermal deposition of inorganic nanostructures on zeolite crystals for mixed matrix membrane fabrication. J. Am. Chem. Soc. 131, 14662-14663 (2009).

10. Chung, T.-S., Jiang, L. Y., Li, Y. \& Kulprathipanja, S. Mixed matrix membranes (MMMs) comprising organic polymers with dispersed inorganic fillers for gas separation. Prog. Polym. Sci. 32, 483-507 (2007). 
11. Liu, G., Xiangli, F., Wei, W., Liu, S. \& Jin, W. Improved performance of PDMS/ceramic composite pervaporation membranes by ZSM-5 homogeneously dispersed in PDMS via a surface graft/coating approach. Chem. Eng. J. 174, 495-503 (2011).

12. Nugent, $\mathrm{P}$. et al. Porous materials with optimal adsorption thermodynamics and kinetics for $\mathrm{CO}_{2}$ separation. Nature 495, 80-84 (2013).

13. Cadiau, A., Adil, K., Bhatt, P. M., Belmabkhout, Y. \& Eddaoudi, M. A metal-organic framework-based splitter for separating propylene from propane. Science 353, 137-140 (2016).

14. Cui, X. et al. Pore chemistry and size control in hybrid porous materials for acetylene capture from ethylene. Science 353, 141-144 (2016).

15. Cadiau, A. et al. Hydrolytically stable fluorinated metal-organic frameworks for energy-efficient dehydration. Science 356, 731-735 (2017).

16. O'Keeffe, M. \& Yaghi, O. M. Deconstructing the crystal structures of metal-organic frameworks and related materials into their underlying nets. Chem. Rev. 112, 675-702 (2012).

17. Eddaoudi, M. et al. Systematic design of pore size and functionality in isoreticular MOFs and their application in methane storage. Science 295, 469-472 (2002).

18. Peng, Y. et al. Metal-organic framework nanosheets as building blocks for molecular sieving membranes. Science 346, 1356-1359 (2014).

19. Bae, T.-H. et al. A high-performance gas-separation membrane containing submicrometer-sized metal-organic framework crystals. Angew. Chem. Int. Ed. 49, 9863-9866 (2010). 
20. Rodenas, T. et al. Metal-organic framework nanosheets in polymer composite materials for gas separation. Nat Mater 14, 48-55 (2015).

21. Al-Maythalony, B. A. et al. Quest for anionic MOF membranes: Continuous sod-ZMOF membrane with $\mathrm{CO}_{2}$ adsorption-driven selectivity. J. Am. Chem. Soc. 137, 1754-1757 (2015).

22. Shen, J. et al. UiO-66-polyether block amide mixed matrix membranes for $\mathrm{CO}_{2}$ separation. J. Memb. Sci. 513, 155165 (2016).

23. Zhang, C., Dai, Y., Johnson, J. R., Karvan, O. \& Koros, W. J. High performance ZIF-8/6FDA-DAM mixed matrix membrane for propylene/propane separations. J. Memb. Sci. 389, 34-42 (2012).

24. Brown, A. J. et al. Interfacial microfluidic processing of metal-organic framework hollow fiber membranes. Science 345, 72-75 (2014).

25. Bachman, J. E., Smith, Z. P., Li, T., Xu, T. \& Long, J. R. Enhanced ethylene separation and plasticization resistance in polymer membranes incorporating metal-organic framework nanocrystals. Nat Mater 15, 845-849 (2016).

26. Knebel, A. et al. Defibrillation of soft porous metal-organic frameworks with electric fields. Science 358, 347-351 (2017).

27. Adil, K. et al. Gas/vapour separation using ultra-microporous metal-organic frameworks: insights into the structure/separation relationship. Chem. Soc. Rev. 46, 3402-3430 (2017).

28. Belmabkhout, Y. et al. Metal-organic frameworks to satisfy gas upgrading demands: fine-tuning the soc-MOF platform for the operative removal of $\mathrm{H}_{2}$ S. J. Mater. Chem. A 5, 3293-3303 (2017). 
29. Assen, A. H. et al. Ultra-tuning of the rare-earth fcu-MOF aperture size for selective molecular exclusion of branched paraffins. Angew. Chem. Int. Ed. 54, 14353-14358 (2015).

30. Qiu, S., Xue, M. \& Zhu, G. Metal-organic framework membranes: from synthesis to separation application. Chem. Soc. Rev. 43, 6116-6140 (2014).

31. Park, K. S. et al. Exceptional chemical and thermal stability of zeolitic imidazolate frameworks. Proc. Natl Acad. Sci. USA 103, 10186-10191 (2006).

32. Chui, S. S.-Y., Lo, S. M.-F., Charmant, J. P. H., Orpen, A. G. \& Williams, I. D. A chemically functionalizable nanoporous material $\left[\mathrm{Cu}_{3}(\mathrm{TMA})_{2}\left(\mathrm{H}_{2} \mathrm{O}\right)_{3}\right]_{\mathrm{n}}$. Science 283, 1148-1150 (1999).

33. Loiseau, T. et al. A rationale for the large breathing of the porous aluminum terephthalate (MIL-53) upon hydration. Chem. Eur. J. 10, 1373-1382 (2004).

34. Rosi, N. L. et al. Hydrogen storage in microporous metal-organic frameworks. Science 300, 1127-1129 (2003).

35. Rosi, N. L. et al. Rod packings and metal-organic frameworks constructed from rod-shaped secondary building units. J. Am. Chem. Soc. 127, 1504-1518 (2005).

36. Xue, D.-X. et al. Tunable rare-earth fcu-MOFs: A platform for systematic enhancement of $\mathrm{CO}_{2}$ adsorption energetics and uptake. J. Am. Chem. Soc. 135, 7660-7667 (2013).

37. Xue, D.-X. et al. Tunable rare earth fcu-MOF platform: access to adsorption kinetics driven gas/vapor separations via pore size contraction. J. Am. Chem.l Soc. 137, 5034-5040 (2015).

38. Zhang, C. et al. Highly scalable ZIF-based mixed-matrix hollow fiber membranes for advanced hydrocarbon separations. AIChE J. 60, 2625-2635 (2014). 
39. Moore, T. T. \& Koros, W. J. Non-ideal effects in organic-inorganic materials for gas separation membranes. J. Mol. Struct. 739, 87-98 (2005).

40. Jia, M., Peinemann, K.-V. \& Behling, R.-D. Molecular sieving effect of the zeolite-filled silicone rubber membranes in gas permeation. J. Memb. Sci. 57, 289-292 (1991).

41. Woo, M., Choi, J. \& Tsapatsis, M. Poly(1-trimethylsilyl-1-propyne)/MFI composite membranes for butane separations. Microporous Mesoporous Mater. 110, 330-338 (2008).

42. Kraftschik, B., Koros, W. J., Johnson, J. R. \& Karvan, O. Dense film polyimide membranes for aggressive sour gas feed separations. J. Memb. Sci. 428, 608-619 (2013).

43. Chatterjee, G., Houde, A. A. \& Stern, S. A. Poly(ether urethane) and poly(ether urethane urea) membranes with high $\mathrm{H}_{2} \mathrm{~S} / \mathrm{CH}_{4}$ selectivity. J. Memb. Sci. 135, 99-106 (1997).

44. Rangnekar, N., Mittal, N., Elyassi, B., Caro, J. \& Tsapatsis, M. Zeolite membranes - a review and comparison with MOFs. Chem. Soc. Rev. 44, 7128-7154 (2015).

45. Wijmans, J. G. \& Baker, R. W. The solution-diffusion model: a review. J. Memb. Sci. 107, 1-21 (1995).

46. Merkel, T. C. et al. Ultrapermeable, reverse-selective nanocomposite membranes. Science 296, 519-522 (2002).

47. Zhang, C. et al. Unexpected molecular sieving properties of zeolitic imidazolate framework-8. J. Phys. Chem. Lett. 3, 2130-2134 (2012).

48. Rungta, M. et al. Carbon molecular sieve structure development and membrane performance relationships. Carbon 115, 237-248 (2017). 
49. Wind, J. D. et al. Relaxation dynamics of $\mathrm{CO}_{2}$ diffusion, sorption, and polymer swelling for plasticized polyimide pembranes. Macromolecules 36, 6442-6448 (2003).

50. Liu, G. et al. Molecularly designed stabilized asymmetric hollow fiber membranes for aggressive natural gas separation. Angew. Chem. Int.l Ed. 55, 13754-13758 (2016).

51. Robeson, L. M. The upper bound revisited. J. Memb. Sci. 320, 390-400 (2008).

52. Koros, W. J. \& Paul, D. R. Design considerations for measurement of gas sorption in polymers by pressure decay. $J$. Polym. Sci. Polym. Phys. Ed. 14, 1903-1907 (1976).

53. Ruthven, D. M. Sorption kinetics for diffusion-controlled systems with a strongly concentration-dependent diffusivity. Chem. Eng. Sci. 59, 4531-4545 (2004).

54. Crank, J. The Mathematics of Diffusion. (Oxford University Press, Oxford, 1979). 